\title{
ASO Author Reflections: Leveraging Real-World Data to Guide Surgical Risk Stratification in Patients Recovered from COVID-19
}

\author{
Anai N. Kothari, MD, MS ${ }^{1}$, and George J. Chang, MD, $\mathbf{M S}^{2}$ \\ ${ }^{1}$ Department of Surgical Oncology, University of Texas MD Anderson Cancer Center, Houston, TX; ${ }^{2}$ Department of Colon \\ and Rectal Surgery, University of Texas MD Anderson Cancer Center, Houston, TX
}

\section{PAST}

The coronavirus disease 2019 (COVID-19) pandemic has created unprecedented and unexpected clinical considerations across multiple treatment settings. This includes determining how, or if, surgical care should be modified in patients undergoing elective surgery after recovering from severe acute respiratory syndrome coronavirus 2 (SARSCoV-2). ${ }^{1}$ Increasingly, it is being recognized that some patients who recover from an acute SARS-CoV-2 infection develop a persistent, longer-term syndrome ("longCOVID") that is associated with an increased risk of thromboembolic events, myocarditis, and pulmonary disorders. ${ }^{2}$ Each of these sequelae may modify surgical risk in the perioperative setting. One proposed solution is delaying surgical treatment until the postoperative risk, especially for patients undergoing elective surgery, returns to baseline. Based on data from other viral syndromes and preliminary results from a large, multinational collaborative, initial recommendations ranged from 4 to 7 weeks to delay elective surgery in those recovered from SARS-CoV2. ${ }^{3}$ However, for time-sensitive conditions including cancer-related surgery, a shorter postponement period may be required, and quantifying the risk in this population is crucial.

\section{PRESENT}

In patients recovering from mild-to-moderate SARSCoV-2 infection, we demonstrated that a wait period of 20 days to perform elective cancer-related surgery appears to be safe. ${ }^{4}$ Using real-world data from our institution, we identified no difference in postoperative readmission, pneumonia, cardiac injury, or thromboembolic events for those recently recovered from SARS-CoV-2 when compared with matched controls. Measuring differences in more specific perioperative parameters, including intraoperative $\mathrm{FiO}_{2}$, average postoperative oxygen saturation, and use of oxygen supplementation (including device), allowed unique insight into the potential physiologic changes related to recent SARS-CoV-2 recovery. From an epidemiologic standpoint, the first patients who underwent surgery after recovering from SARS-CoV-2 infection were at our institution in May 2020. Between May 2020 and October 2020, approximately $2.0 \%$ of elective surgical procedures were performed on patients with prior SARS$\mathrm{CoV}-2$ infection. This percentage increased over the study period and highlights the need for continued close attention to surgical outcomes in this population. For patients recovered from mild-to-moderate SARS-CoV-2 infection, these data should be used to inform consensus guidelines that guide patients and practioners on when it is safe to proceed with lower-risk, elective cancer-related surgery.

\section{FUTURE}

Given the dynamic nature of the COVID-19 pandemic, leveraging real-world data to rapidly generate real-world evidence has proved to be invaluable. At MD Anderson, the Data-Driven Determinants for COVID-19 Oncology Discovery (D3CODE) group has facilitated this by aggregating multiple electronic data streams onto a single platform. This includes electronic health records,
(C) Society of Surgical Oncology 2021

First Received: 15 June 2021

Accepted: 15 June 2021;

Published Online: 24 July 2021

G. J. Chang, MD, MS

e-mail: gchang@mdanderson.org 
anesthesia and intraoperative data, and other structured and unstructured clinical, basic, and translational data sources. ${ }^{5}$ Considering the worldwide burden of SARS-CoV-2 infection, the wide range of clinical presentations, and the variation in persistent symptoms, continuing to effectively use real-world data to guide surgical treatment in response to the pandemic will remain critical. This will include, but is not limited to, prediction of surgical risk in patients with "long-COVID," recovering from more severe SARS-CoV2 infections, and developing tools to more quickly generate evidence as new, potentially unanticipated clinical questions arise as we move from a pandemic to postpandemic phase.

DISCLOSURES George Chang, Consultant: Medicaroid, Advisory board: 11Health, Advisory board: Genomic Health.

\section{REFERENCES}

1. Baiocchi G, Aguiar S Jr, Duprat JP, et al. Early postoperative outcomes among patients with delayed surgeries after preoperative positive test for SARS-CoV-2: a case-control study from a single institution. J Surg Oncol. 2021. https://doi.org/10.1002/jso.26377.

2. Mahase E. Covid-19: what do we know about "long covid"? BMJ. 2020;370:m2815.

3. COVIDSurg Collaborative GC. Timing of surgery following SARS-CoV-2 infection: an international prospective cohort study. Anaesthesia. 2021;76(6):748-58.

4. Kothari AN, DiBrito SR, Lee JJ, et al. Surgical outcomes in cancer patients undergoing elective surgery after recovering from mild-tomoderate SARS-CoV-2 infection [published online ahead of print, 2021 Jun 26]. Ann Surg Oncol. 2021;1-8. https://doi.org/10.1245/ s10434-021-10291-9

5. Alfaro-Munoz K, Hallatt G, Sookprasong J, et al. Building a data foundation: how MD Anderson and Palantir are partnering to accelerate research and improve patient care. J Clin Oncol. 2019;37(15_suppl):e18077-e18077.

Publisher's Note Springer Nature remains neutral with regard to jurisdictional claims in published maps and institutional affiliations. 